\title{
Low-Field Magnetic Resonance Imaging of the Thumb Base in Severe Symptomatic Osteoarthritis: A Comparison with Radiographs and Clini- cal Symptoms
}

\author{
Gudmundur J. Eliasson ${ }^{*, 1}$, Eythor Bjorgvinsson ${ }^{2}$ and Helgi Jonsson*,3 \\ ${ }^{I}$ Roentgen Domus Medica and Landspitalinn University Hospital Reykjavik, Iceland \\ ${ }^{2}$ Roentgen Domus Medica, Reykjavik, Iceland \\ ${ }^{3}$ Landspitalinn University Hospital Reykjavik Iceland
}

\begin{abstract}
The objectives of this pilot study were to assess the findings of low-field MRI in symptomatic thumb base osteoarthritis (OA), to identify suitable MRI sequences with or without Gadolinium contrast, and to compare these findings with conventional radiographs scored by the Altman atlas and patient reported pain.

Twelve patients with symptomatic thumb base osteoarthritis participated. We used a 0,2T MRI system, before and after i.v. Gadolinium. The MRI images were scored on a 0-3 scale for synovitis (SYN), bone marrow edema (BME), cysts/erosions (CE) and osteophytes (OP).

Interreader agreement on MRI scores was best for BME and CE but less for SYN and OP. Difficulties were in determining the soft tissue involvement of the CMC1 joint, especially in demarcating the joint capsule and ligament pathology. The most informative MRI sequences were the High Resolution Gradient Echo, the STIR and the 3D volume.Gadolinium contrast provided little additional information.

Cumulative MRI and radiographic scores for the $24 \mathrm{CMC} 1$ joints studied were strongly correlated $\left(\mathrm{R}_{\mathrm{s}} 0.81, \mathrm{p}<0.001\right)$. Both BME in adjacent bones and SYN in the CMC1 joint correlated with rest pain $\left(\mathrm{R}_{\mathrm{s}} 0.54, \mathrm{p}<0.01\right.$ and $\left.\mathrm{R}_{\mathrm{s}} 0.45, \mathrm{p}<0.05\right)$ respectively). BME was also associated with pain intensity on a $0-10$ scale ( $\mathrm{R} 0.44, \mathrm{p}<0.05)$. Other radiographic and MRI variables showed no association with pain.

Low-field MRI reflects CMC1 joint osteoarthritis damage and gives additional information regarding bone marrow edema and synovitis, both of which are strongly related to clinical symptoms. This study encourages further research to identify the most suitable equipment and sequences and to standardize the reading of MRI images.
\end{abstract}

Keywords: Magnetic resonance imaging, Hand osteoarthritis, Synovitis, Bone marrow edema.

\section{BACKGROUND}

Osteoarthritis $(\mathrm{OA})$ of the thumb base is a common and disabling form of osteoarthritis [1-3]. It is characterized by a slow variable course and sometimes by episodes with severe symptoms. Standard radiographs are considered the gold standard for the diagnosis of this condition [4], but they have serious limitations, mainly by not reflecting disease activity and thus the relation between radiologic changes and symptoms is generally poor [5]. Furthermore, the slow radiographic progression of $\mathrm{OA}$ hampers therapeutic studies which have to go on for years to register effects on disease progression. More dynamic imaging is needed to reflect the ongoing disease progress and both scintigraphy and ultrasound have been advocated in this respect [6,7].

Magnetic resonance imaging (MRI) has been extensively studied in knee OA. It has several advantages over radiographs in the diagnosis and understanding the disease proc-

*Address correspondence to these authors at the Roentgen Domus Medica, Egilsgata 3, IS-101 Reykjavik, Iceland; E-mail: gje @ simnet.is

Department of Rheumatology, Landspitalinn University Hospital, IS-108 Reykjavik, Iceland; E-mail: helgijon@landspitali.is ess. Better imaging of soft tissues such as cartilage, ligaments and joint capsule along with inflammatory and reactive changes in the joints and bones has wastly improved our understanding of the disease process. "Bone marrow edema" (BME) in particular has been found to associate with symptoms and progression of knee OA [8].

MRI imaging of the hands in rheumatoid arthritis is now gaining acceptance as a valuable tool to assess prognosis and monitor the disease progress $[9,10]$. We are not aware of previous studies using MRI in thumb base OA.

The disadvantages of MRI imaging include cost, availability, long examination times and most of all, difficulty in reading and interpretation of the findings. We report here a pilot study aiming at determining the most suitable low-field MRI sequences for the assessment of thumb base OA comparing them with symptoms and conventional radiographs. The MRI equipment chosen was an office-based 0,2T system with and without the use of gadolinium contrast.

\section{PATIENTS AND METHODS}

Twelve consecutive hand OA patients presenting with clinical symptomatic thumb base osteoarthritis based on 
squaring and suluxation of that joint in the absence of other rheumatological diagnoses were included in the study. An additional requirement was that the symptoms were predominantly unilateral and the reported pain in the symptomatic joint for the last 48 hours had to be at least 5 on an 0-10 scale. After informed consent, they registered pain on a 0 -10 scale in both thumb bases and reported on the presence or absence of rest pain. There were 11 females and 1 male, median age was 57, range 45-71. One of the patients developed a contralateral flare in symptoms while waiting for MRI and thus reported equal pain on both sides. Rest pain was reported by 8 patients, unilateral in all cases.

All patients had standard digital radiographs of the hands. They were scored by both radiologists (GJE,EB) according to the Altman atlas [4], scoring osteophytes (0-3), joint space narrowing (0-3) and erosions (0-3). All readings of radiographs were performed blindly.

MRI imaging was done by a 0,2T Artoscan-Dedicated MRI system from ESAOTE, Italy. The coil used was a dual phased array wrist coil. The MRI sequences were all in the coronal plane; High Resolution Gradient Echo (HRGE) (TR580, TE16), Gradient Echo STIR (TR1120, TE16), Turbo 3D T1 (TR40, TE16) and Spin Echo T1 (TR600, TE26). The field of view was $12 \mathrm{~cm}$, interspace $2 \mathrm{~mm}$, section thickness $3 \mathrm{~mm}$ for all sequences except $0.6 \mathrm{~mm}$ for $3 \mathrm{D} \mathrm{T} 1$. The imaging display matrix was 512 x 512 for HRGE and $256 \times 256$ for the other sequences The more symptomatic hand had $\mathrm{T} 1$ sequences before and after $10 \mathrm{ml}$ i.v. Gadolinium.

The MRI images of the CMC1 joints were scored on a 0 3 scale for synovitis (SYN) (based on capsular hypertrophy and joint effusion), bone marrow edema (BME), cysts/ erosions (CE) and osteophytes (OP). Due to difficulties in measuring SYN quantitatively, an additional measure of right-left comparison was added. Consensus scores for both radiographs and MRI images were reached at a second sitting.

The study was approved by the Icelandic National Ethics Committee. a

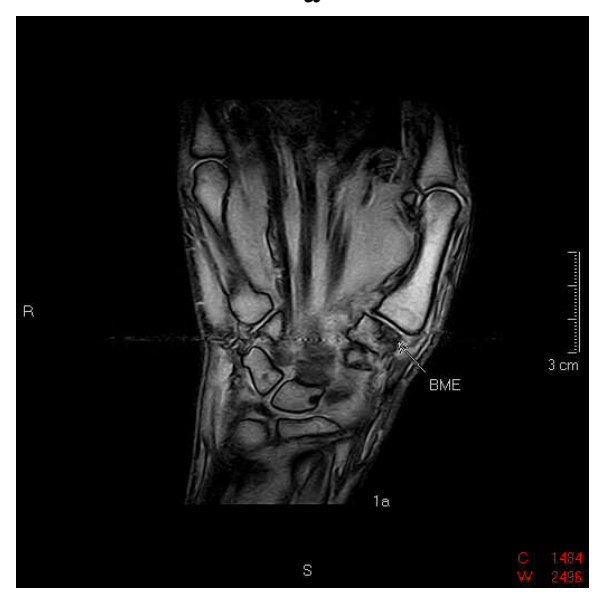

b

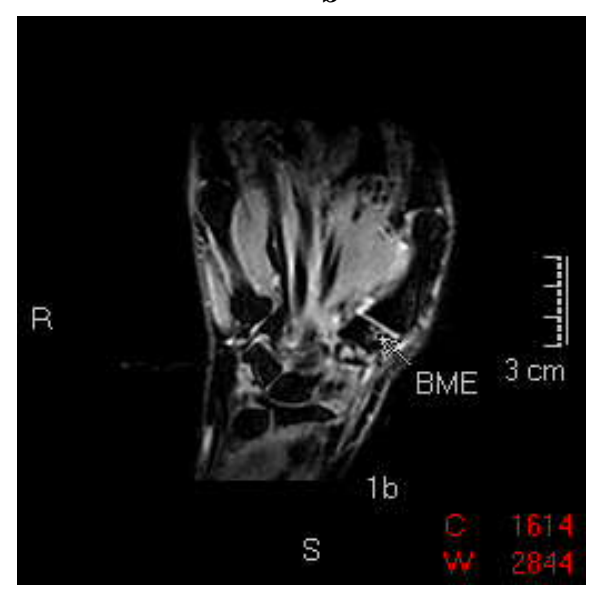

c

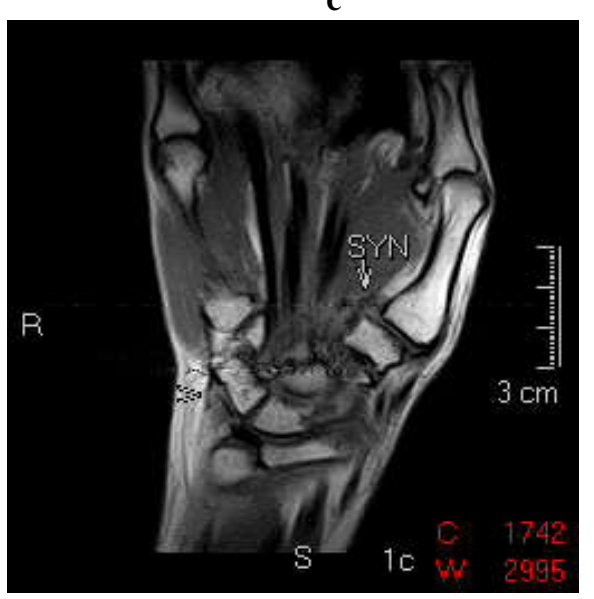

d

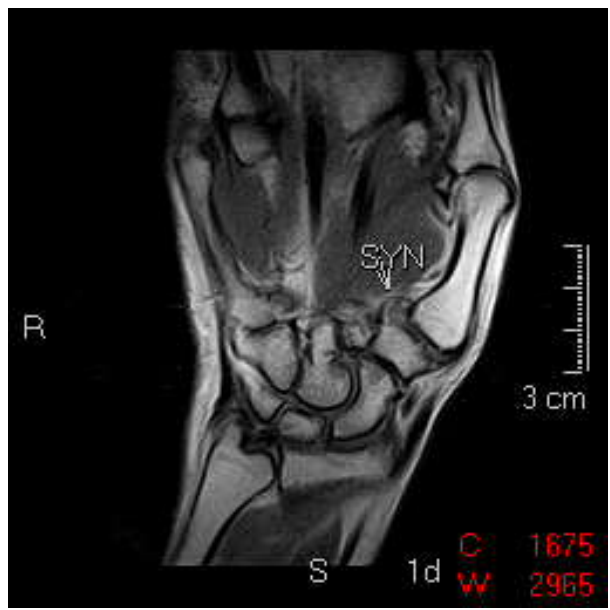

e

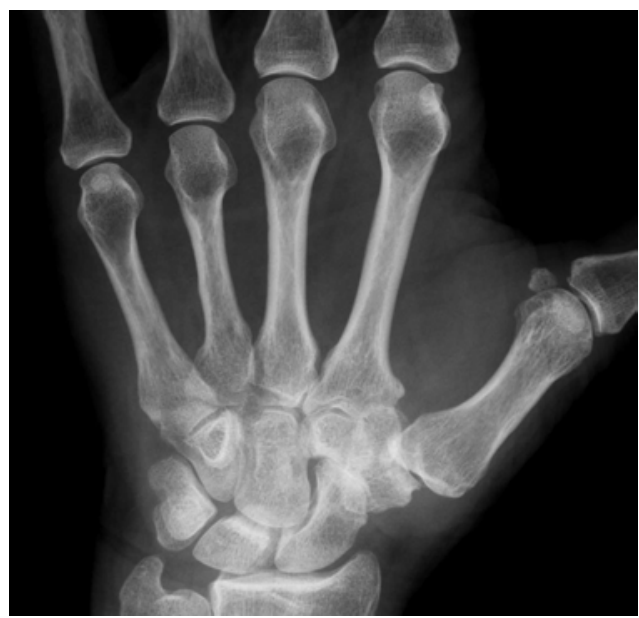

Fig. (1). Examples of thumb base MRI images.
a) HRGE
b) STIR
c) T1 before gadolinium
d) $\mathrm{T} 1$ after gadolinium
e) Digital radiograph. 


\section{Statistics}

All $24 \mathrm{CMC} 1$ joints were used for statistical calculations, comparing the joint with predominant symptoms with the less symptomatic side. The intra-class coefficient (ICC) was used to assess interreader agreement., the Spearman rank test $\left(\mathrm{R}_{\mathrm{s}}\right)$ for correlation and chi-square for comparison between groups. Multivariate analysis was done by backwards linear regression.

\section{RESULTS}

Interreader agreement on MRI scores of the $\mathrm{CMC1}$ joint varied considerably and was acceptable for BME (ICC 0.88) and CE (ICC 0.88) but less so for SYN (ICC 0.63) and OP (ICC 0.66). In the case of SYN, all 24 joints examined had some degree of synovial reactive changes, but there were reading difficulties in determining the soft tissue involvement of the $\mathrm{CMC} 1$ joint, especially demarcating the joint capsule and ligament pathology.
For best anatomical-pathological visualisation we found the most informative MRI sequences in this study to be the HRGE, STIR and the 3D T1 volume. HRGE has the best resolution, STIR is essential to demonstrate bone marrow edema and the 3D T1 volume allows evaluations in all planes and visualisation of smaller structures such as osteophytes and cysts. Ordinary dose of Gadolinium contrast provided little additional information. Chosen examples of MRI sequences from two joints are shown below (Figs. 1 and 2).

On comparison between MRI and radiographic scores, there was little correlation between the four MRI variables and the three variables scored on the radiographs. On the other hand, aggregate MRI scores showed a very high degree of correlation with aggregate radiography scores for the CMC1 joint $\left(\mathrm{R}_{\mathrm{s}} 0.81, \mathrm{p}<0.001\right)($ Fig. 3). The aggregate scores did not show any significant association with symptoms. A summary of radiographic and MRI scores is given in Table 1.

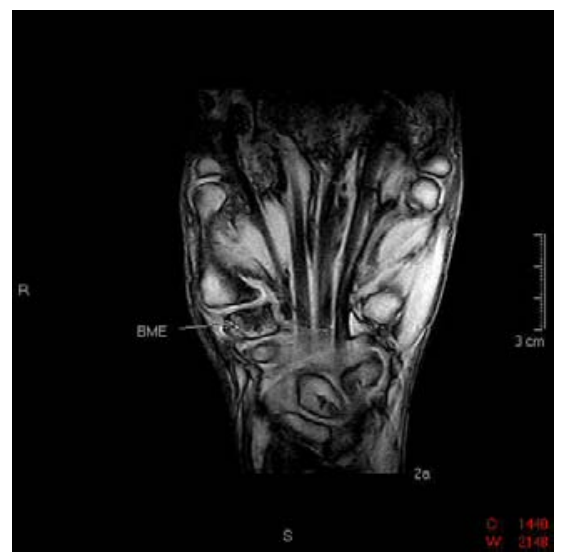

b

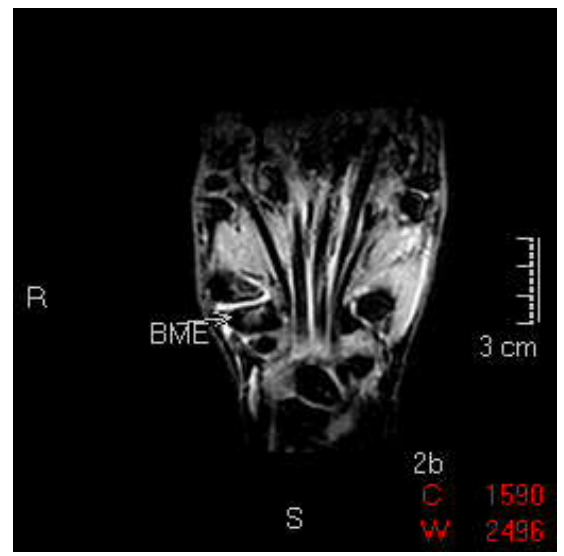

c

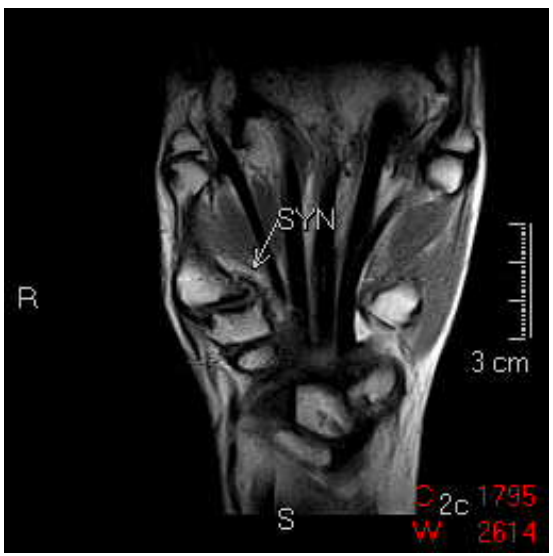

d

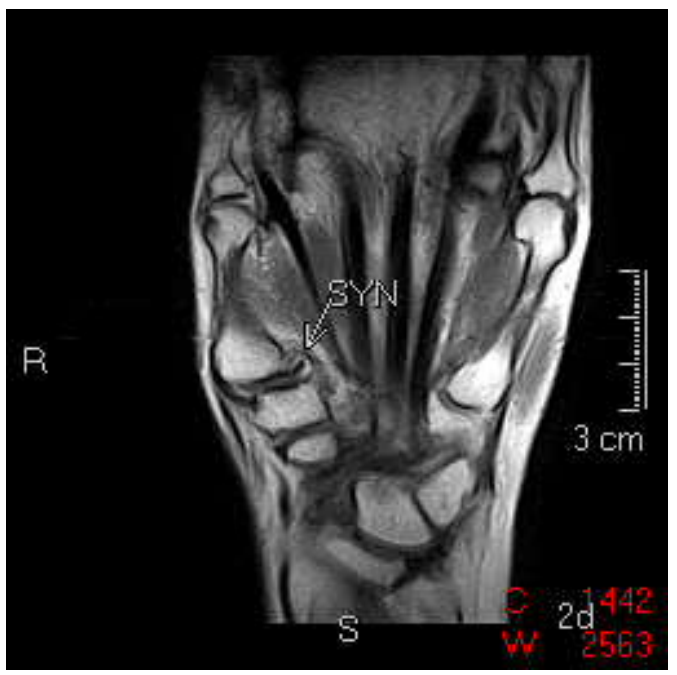

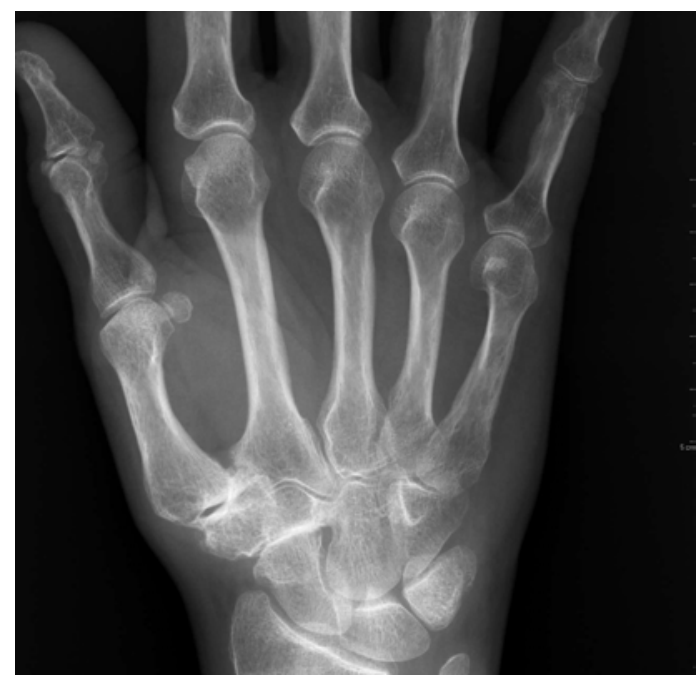

Fig. (2). Examples of thumb base MRI images.
a) HRGE
b) STIR
c) $\mathrm{T} 1$ before gadolinium
d) $\mathrm{T} 1$ after gadolinium
e) Digital radiograph. 


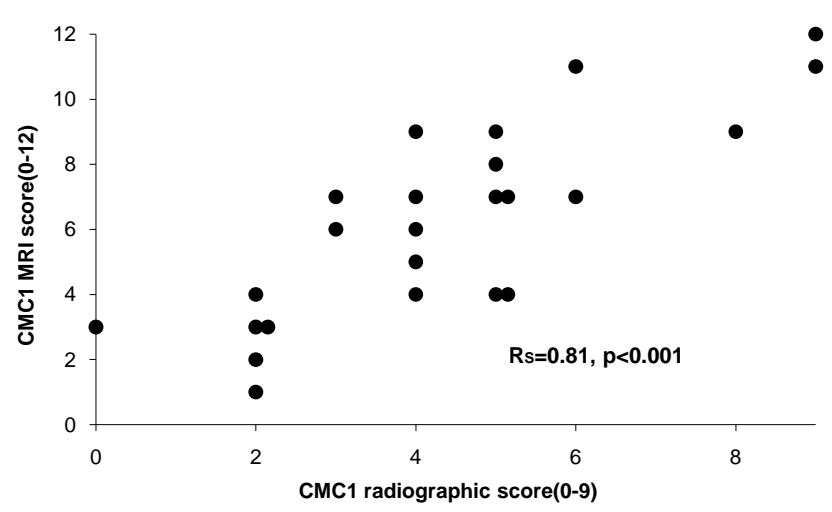

Fig. (3). The relationship between of aggregate $\mathrm{CMC} 1$ joint scores from radiographs and MRI.

On bivariate analysis of the relation between MRI imaging and symptoms, both BME $\left(\mathrm{R}_{\mathrm{s}} 0.54, \mathrm{p}<0.01\right)$ (Fig. 4) and SYN $\left(R_{\mathrm{s}} 0.45, \mathrm{p}<0.05\right)$ correlated significantly with rest pain and BME also correlated with pain intensity $\left(R_{s} 0.44\right.$, $\mathrm{p}<0.05$ ) (Fig. 5). The only patient who had a BME score of 3 in the absence of rest pain had more BME on the other side and reported rest pain only on that side. Odds ratios for the co-occurrence of rest pain and BME were clearly significant even with different cut-off points for BME. (BME $\geq 1$ vs. rest pain; OR 11.7 (1.1-120), $\mathrm{p}<0.05, \mathrm{BME} \geq 2$ vs. rest pain: OR 11.7 (1.5-92), $\mathrm{p}<0.05)$. On multivariate analysis however, the independent effect of SYN on rest pain did not reach significance due to its association with BME. However, ten of the eleven patients who reported more symptoms on one side had more SYN on the painful side $(\mathrm{p}<0.01)$ although it did not register as a difference on the 0-3 scale. Neither CE nor OP showed a significant relation to pain nor did any of the radiographic scores.

\section{DISCUSSION}

In this pilot study of $24 \mathrm{CMC} 1$ joints in 12 patients with severe symptomatic thumb base osteoarthritis, scores from MRI images showed a significant association with patient reported pain. No such association was seen with conventional radiographs. The presence of bone marrow edema in particular showed a clear relation to both rest pain and pain severity. Synovitis was also associated with symptoms, but quantitation of synovitis proved problematic with our equipment, despite the use of gadolinium contrast.

One of our aims was to determine the most useful lowfield MRI sequences, thus optimizing the use of the equipment and shortening examination times. The ideal situation for clinical use would be having a non-invasive standard run of a few chosen sequences without contrast and a rapid reading protocol. We had planned to extend this study, investigating a larger group of patients with a modified protocol, using only selected sequences. However, the problems of quantifying synovitis indicated a need for a different protocol. Doubling the dose of gadolinium contrast has been shown to improve clarity of synovial tissues [11], but this increases the cost of the examination considerably. Instead, we intend to use higher resolution MRI equipment aiming for increased resolution of soft tissues and cartilage, preferably without the use of contrast to minimize patient discomfort and to shorten the time of aquisition.

Severe symptomatic flares of thumb base OA are fairly common in the clinical situation. Despite that, optimal treatment is in dispute. Intraarticular glucocorticoid injections are widely used and recommended in the European League against Rheumatism (EULAR) guidelines for the treatment of hand OA [12]. This is in spite of a recent placebo controlled study showing no effect of such treatment [13]. In an interesting study of which MRI findings predicted a favor-

Table 1. MRI and Radiographic Scores in the CMC1 Joints in Relation to Symptoms

\begin{tabular}{|c|c|c|c|c|}
\hline & All Joints $(n=24)$ & $\begin{array}{l}\text { Predominant Side } \\
\qquad(\mathbf{n}=\mathbf{1 1})^{*}\end{array}$ & $\begin{array}{c}\text { Less Symptomatic } \\
\text { Side }(n=11)^{*}\end{array}$ & Rest Pain $(n=8)$ \\
\hline & Median(range) & Median(range) & Median(range) & Median(range) \\
\hline Pain intensity (0-10) & $5(0-10)$ & $8(5-10)$ & $3(0-4)$ & $7.5(6-10)$ \\
\hline \multicolumn{5}{|l|}{ MRI scoring } \\
\hline $\operatorname{BME}(0-3)$ & $1(0-3)$ & $2(0-3)$ & $0(0-3)$ & $2(0-3)$ \\
\hline SYN (0-3) & $2(1-3)$ & $3(2-3)$ & $2(1-3)$ & $3(2-3)$ \\
\hline OP $(0-3)$ & $2(0-3)$ & $2(0-3)$ & $2(0-3)$ & $2(0-3)$ \\
\hline CE $(0-3)$ & $1(0-3)$ & $1(0-2)$ & $1(0-3)$ & $1.5(0-2)$ \\
\hline Aggregate (0-12) & $6.5(1-12)$ & $7(3-11)$ & $4(1-12)$ & $8(3-11)$ \\
\hline \multicolumn{5}{|l|}{ Radiograph scoring } \\
\hline Osteophytes (0-3) & $2(0-3)$ & $2(0-3)$ & $1(1-3)$ & $2(0-3)$ \\
\hline Joint space narrowing (0-3) & $1(0-3)$ & $2(0-3)$ & $1(1-3)$ & $1.5(0-3)$ \\
\hline Erosions $(0-3)$ & $1(0-3)$ & $1(0-3)$ & $1(0-3)$ & $1.5(0-3)$ \\
\hline Aggregate (0-9) & $4(0-9)$ & $5(0-9)$ & $3(2-9)$ & $5(0-9)$ \\
\hline
\end{tabular}

* One patient reported equal symptoms on both sides. 


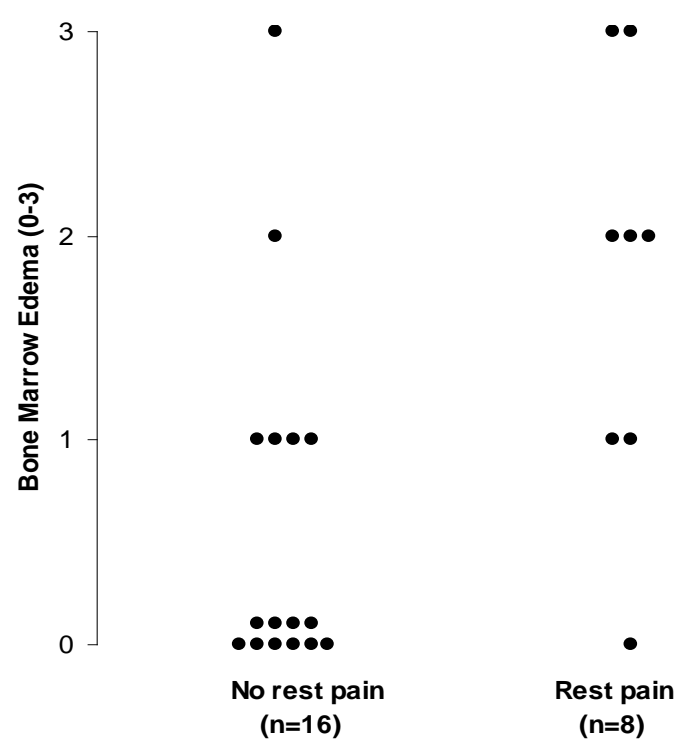

Fig. (4). Patient reported rest pain in relation to bone marrow edema.

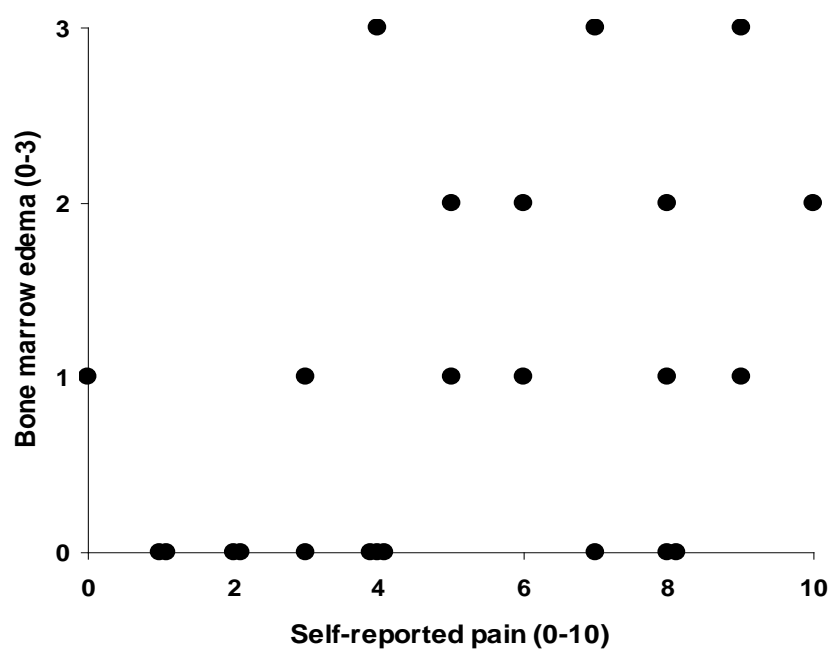

Fig. (5). Patient reported pain severity in relation to bone marrow edema $\left(\mathrm{R}_{\mathrm{s}} 0,44, \mathrm{p}<0.05\right)$.

able effect of glucocorticoid injections in the acromioclavicular joint, the presence of synovitis was the best predictor of response [14]. It is easy to postulate that the information from MRI imaging may affect the therepeutic approach to symptomatic thumb base OA; synovitis may well benefit from local injections, whereas bone marrow edema could require more bone directed treatment, local or systemic. We are also in possession of data indicating that MRI images are useful in studies of such therapeutic interventions [15].

The main limitations of this study was naturally its small size and the use of statistical methods on bilateral joints. The statistical results have therefore to be interpreted with great caution.

Finally, we conclude that low-field MRI is a very promising method for the assessment of thumb base OA. The information regarding bone marrow edema and synovitis adds a new dimension to the understanding of pain in the CMC1 joints, particularly as these conditions may warrant different therapeutic approaches. This study encourages further research to identify the most suitable equipment and sequences and to standardize the reading of the MRI images.

\section{ACKNOWLEDGEMENTS}

This work was supported by Roentgen Domus Medica, the University of Iceland Research Fund and the Icelandic Osteoarthritis Research Fund.

Thanks to: Our participants for their patience and to radiographers Gudrun Fridriksdottir and Thuridur Halldorsdottir.

\section{REFERENCES}

[1] Dillon CF, Hirsch R, Rasch EK, Gu Q. Symptomatic hand osteoarthritis in the United States: prevalence and functional impairment estimates from the third U.S. National Health and Nutrition Examination Survey, 1991-1994. Am J Phys Med Rehabil 2007; 86: $12-21$.

[2] Fautrel B, Hilliquin P, Rozenberg S, et al. Impact of osteoarthritis: results of a nationwide survey of 10.000 patients consulting for OA. Joint Bone Spine 2005; 72: 235-40.

[3] Dominick KL, Jordan JM, Renner JB, Kraus VB. Relationship of radiographic and clinical variables to pinch and grip strength among individuals with osteoarthritis. Arthritis Rheum 2005; 52: 1424-30.

[4] Altman RD, Hochberg M, Murphy WA Jr, Wolfe F, Lequesne M. Atlas of individual radiographic features in osteoarthritis. Osteoarthritis Cart 1995; 3(Suppl A): 3-70.

[5] Dahaghin S, Bierma-Zeinstra SM, Reijman M, Pols HA, Hazes JM, Koes BW. Prevalence and determinants of one month hand pain and hand related disability in the elderly (Rotterdam study). Ann Rheum Dis 2005; 64: 99-104.

[6] Jonsson H, Eliasson GJ, Petursson E. Scintigraphic hand osteoarthritis (OA)--prevalence, joint distribution, and association with OA at other sites. J Rheumatol 1999; 26: 1550-6.

[7] Iagnocco A, Coari G. Usefulness of high resolution US in the evaluation of effusion in osteoarthritic first carpometacarpal joint. Scand J Rheumatol 2000; 29: 170-3.

[8] Hunter DJ, Zhang Y, Niu J, et al. Increase in bone marrow lesions associated with cartilage loss: a longitudinal magnetic resonance imaging study of knee osteoarthritis. Arthritis Rheum 2006; 54: 1529-35.

[9] Scheel AK, Hermann KG, Ohrndorf S, et al. Prospective 7 year follow up imaging study comparing radiography, ultrasonography, and magnetic resonance imaging in rheumatoid arthritis finger joints. Ann Rheum Dis 2006; 65: 595-600.

[10] Lindegaard HM, Vallo J, Horslev-Petersen K, Junker P, Ostergaard M. Low-cost, low-field dedicated extremity magnetic resonance imaging in early rheumatoid arthritis: a 1-year follow-up study. Ann Rheum Dis 2006; 65: 1208-12.

[11] Eshed I, Althoff CE, Schink T, et al. Low-field MRI for assessing synovitis in patients with rheumatoid arthritis: Impact of Gd-DTPA dose on synovitis scoring. Scand J Rheumatol 2006; 35: 277-82.

[12] Zhang W, Doherty M, Leeb BF, et al. EULAR evidence based recommendations for the management of hand osteoarthritis: Report of a task force of the EULAR standing committee for international clinical studies including therapeutics (ESCISIT). Ann Rheum Dis 2007; 66: 377-88.

[13] Meenagh GK, Patton J, Kynes C, Wright GD. A randomised controlled trial of intra-articular corticosteroid injection of the carpometacarpal joint of the thumb in osteoarthritis. Ann Rheum Dis 2004; 63: 1260-3.

[14] Strobel K, Pfirrmann CW, Zanetti M, Nagy L, Hodler J. MRI features of the acromioclavicular joint that predict pain relief from intraarticular injection. AJR 2003; 181: 755-60. 
[15] Eliasson GJ, Bragason AO, Bjorgvinsson E, Jonsson H. Low level laser therapy (LLLT) of the osteoarthritic CMC1 joint. Report of six patients using magnetic resonance imaging (MRI) to monitor changes after treatment. Scand J Rheumatol 2006; 35(Suppl 121): 50

Received: September 15, 2008

(C) Eliasson et al.; Licensee Bentham Open.

This is an open access article licensed under the terms of the Creative Commons Attribution Non-Commercial License (http://creativecommons.org/licenses/by-nc/3.0/) which permits unrestricted, non-commercial use, distribution and reproduction in any medium, provided the work is properly cited. 